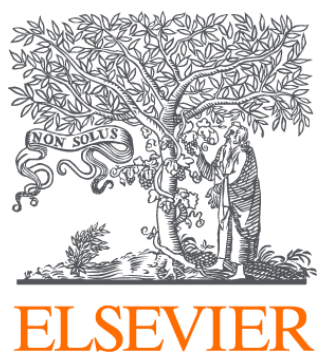

Since January 2020 Elsevier has created a COVID-19 resource centre with free information in English and Mandarin on the novel coronavirus COVID-

19. The COVID-19 resource centre is hosted on Elsevier Connect, the company's public news and information website.

Elsevier hereby grants permission to make all its COVID-19-related research that is available on the COVID-19 resource centre - including this research content - immediately available in PubMed Central and other publicly funded repositories, such as the WHO COVID database with rights for unrestricted research re-use and analyses in any form or by any means with acknowledgement of the original source. These permissions are granted for free by Elsevier for as long as the COVID-19 resource centre remains active. 


\title{
Plasma zinc status and hyperinflammatory syndrome in hospitalized COVID-19 patients: An observational study
}

\author{
Gil Verschelden ${ }^{\mathrm{a}, 1, *}$, Maxim Noeparast ${ }^{\mathrm{b}, \mathrm{c}, 1, *}$, Maryam Noparast ${ }^{\mathrm{d}}$, Mathijs \\ Christiaan Goossens ${ }^{\mathrm{e}}$, Maïlis Lauwers ${ }^{\mathrm{f}}$, Frédéric Cotton ${ }^{\mathrm{f}}$, Charlotte Michel ${ }^{\mathrm{g}}$, Cleo Goyvaerts ${ }^{\mathrm{h}}$, \\ Maya Hites ${ }^{a}$ \\ ${ }^{a}$ Clinic of Infectious Diseases, Cliniques Universitaires de Bruxelles (CUB), Erasme Hospital, Université Libre de Bruxelles (ULB), 1070 Brussels, Belgium \\ ${ }^{\mathrm{b}}$ Institute of Molecular Oncology, Member of the German Center for Lung Research (DZL), Philipps University, 35043 Marburg, Germany \\ ${ }^{\mathrm{c}}$ Fonds Wetenschappelijk Onderzoek (FWO) - Vlaanderen, Belgium \\ ${ }^{\mathrm{d}}$ Department of Urologic Sciences, University of British Columbia, Vancouver, BC, Canada \\ e Vrije Universiteit Brussel, Laarbeeklaan 103, 1090 Brussels, Belgium \\ ${ }^{\mathrm{f}}$ Department of Clinical Chemistry, Laboratoire Hospitalier Universitaire de Bruxelles (LHUB-ULB), Université Libre de Bruxelles (ULB), Brussels, Belgium \\ ${ }^{g}$ Department of Microbiology, Laboratoire Hospitalier Universitaire de Bruxelles (LHUB-ULB), Brussels, Belgium \\ ${ }^{\mathrm{h}}$ Laboratory for Molecular and Cellular Therapy (LMCT), Vrije Universiteit Brussel (VUB), 1090 Brussels, Belgium
}

\section{A R T I C L E I N F O}

\section{Keywords:}

SARS-CoV-2

COVID-19

Zinc

cHIS

Inflammation

\begin{abstract}
A B S T R A C T
Zinc deficiency is associated with impaired antiviral response, cytokine releasing syndrome (CRS), and acute respiratory distress syndrome. Notably, similar complications are being observed during severe SARS-CoV-2 infection.

We conducted a prospective, single-center, observational study in a tertiary university hospital (CUB-Hôpital Erasme, Brussels) to address the zinc status, the association between the plasma zinc concentration, development of CRS, and the clinical outcomes in PCR-confirmed and hospitalized COVID-19 patients.

One hundred and thirty-nine eligible patients were included between May 2020 and November 2020 (median age of 65 years [IQR $=54,77])$.

Our cohort's median plasma zinc concentration was $57 \mu \mathrm{g} / \mathrm{dL}$ (interquartile range $[\mathrm{IQR}]=45,67)$ compared to $74 \mu \mathrm{g} / \mathrm{dL}(\mathrm{IQR}=64,84)$ in the retrospective non-COVID-19 control group $(\mathrm{N}=1513 ; p<0.001)$. Markedly, the absolute majority of COVID-19 patients (96\%) were zinc deficient $(<80 \mu \mathrm{g} / \mathrm{dL})$.

The median zinc concentration was lower in patients with CRS compared to those without CRS (-5 $\mu \mathrm{g} / \mathrm{dL} ; 95 \%$ $\mathrm{CI}=-10.5,0.051 ; p=0.048)$.

Among the tested outcomes, zinc concentration is significantly correlated with only the length of hospital stay (rho $=-0.19 ; p=0.022$ ), but not with mortality or morbidity. As such, our findings do not support the role of zinc as a robust prognostic marker among hospitalized COVID-19 patients who in our cohort presented a high prevalence of zinc deficiency. It might be more beneficial to explore the role of zinc as a biomarker for assessing the risk of developing a tissue-damaging CRS and predicting outcomes in patients diagnosed with COVID-19 at the early stage of the disease.
\end{abstract}

\footnotetext{
Abbreviations: ARDS, Acute respiratory distress syndrome; AUROC, Area under the receiver operating characteristic curve; BMI, Body mass index; cHIS, COVID-

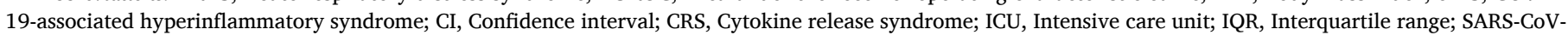
2 , severe acute respiratory syndrome coronavirus 2 .

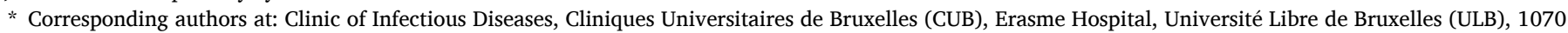

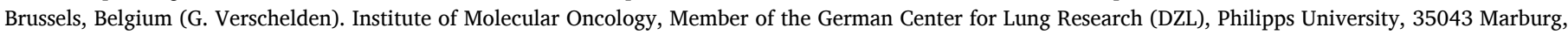
Germany (M. Noeparast).

E-mail addresses: Gil.Verschelden@erasme.ulb.ac.be (G. Verschelden), Maxim.Noeparast@staff.uni-marburg.de (M. Noeparast).

1 Shared first authors, to be cited as Verschelden, Noeparast, et al.
} 


\section{Introduction}

Severe acute respiratory syndrome coronavirus 2 (SARS-CoV-2) is associated with significant mortality and morbidity in a subgroup of patients who develop cytokine release syndrome (CRS) and the related acute respiratory distress syndrome (ARDS) [1,2].

The SARS-CoV-2 point of entry into human lung cells is considered to be Angiotensin-Converting Enzyme 2 (ACE2) receptor [3]. In human lungs, ACE2 receptors are expressed on the surface of surfactant secreting alveolar type II (AT2) cells and pulmonary macrophages [4-6]. AT2 cells, accounting for $\sim 5 \%$ of the human pneumocytes, facilitate the mechanical aspects of lung function by secreting surfactant to ensure the essential elasticity of the alveoli and gas exchange [7-9]. AT2 cells are also suggested to serve as the progenitor pool for the pneumocyte type I cells ( $\sim 95 \%$ of pneumocytes), thereby supporting lung damage repair and protecting it from fibrosis [7-9]. The binding of SARS-CoV-2 to the ACE2 receptor via its spike protein is thought to disrupt ACE2 receptors and the corresponding AT2 cells [9]. Furthermore, ACE2 and another enzyme ACE are both critical effectors of the Renin-Angiotensin System (RAS). RAS exerts its inflammodulatory effects via triggering the synthesis of different angiotensin peptides through two counteracting axes, ACE2 and ACE. ACE2 can counteract the vasoconstrictive and proinflammatory impact of the ACE-AngiotensinII-AT1 receptor axis [9]. As such, SARS-CoV-2 infection leads to lung-damaging complications in a subset of patients, at least partly via targeting the AT2 cells, disrupting the RAS feedback loop, and unleashing Angiotensin II effects $[9,10]$.

Zinc is the second most abundant common trace mineral in humans, yet $\sim 20 \%$ of the global population is estimated to suffer from different degrees of zinc deficiency [11]. Zinc has broad biological functions and has a pivotal role in the immune response [12]. Both ACE2 and ACE enzymatic activities are zinc-dependent, and zinc deficiency negatively affects their kinetics $[10,13]$. It has been hypothesized that zinc deficiency can contribute to ARDS by disrupting ACE2 activity [10]. Although zinc deficiency negatively affects both ACE2 and ACE, it may lead to more harmful consequences in patients with decreased ACE2 levels by mitigating the remaining ACE2 activity.

Given the broad biological functions of zinc, it is no surprise that multiple mechanisms other than RAS-related ones have been proposed through which zinc can affect SARS-CoV-2 and the related immune response. We and others have recently reviewed such evidence [14-16]. Indeed, precedent evidence suggests that zinc deficiency can also be associated with lung-damaging complications such as ARDS [17-20] as well as an impaired antiviral response [11,12,16,21,22]. For instance, zinc deficiency is associated with excessive TNF- $\alpha$ and IL- 6 activity $[23,24]$, factors that have a significant role in CRS [25]. Yet, the zinc status and its association with SARS-CoV-2 needs better clinical elucidation.

Herein, we designed a study to determine the zinc status, explored the association between the plasma zinc concentration, the development of CRS, and the clinical outcomes in hospitalized COVID-19 patients.

\section{Methods}

We conducted a prospective, single-center, observational study in a tertiary university hospital (CUB-Hôpital Erasme, Brussels) between May 2020 and November 2020.

\subsection{Study participants}

Consecutive hospitalized adult patients with PCR-confirmed SARSCoV-2 infection were enrolled after obtaining informed consent. We performed clinical and laboratory assessments within $72 \mathrm{~h}$ of admission. Age, gender, body mass index (BMI), smoking status, medical history, and vital parameters were recorded at the time of inclusion. An atomic absorption spectroscopy technique was used to measure zinc concentrations in the plasma [26].
The severity of COVID-19 was assessed daily during the course of hospitalization using the WHO 10-point system [27]. Individual inflammatory parameters were assessed and summed on the day of patients' study inclusion according to an additive six-point clinical scale developed and validated by Webb et al. [28] to determine the presence and severity of COVID-19-associated hyperinflammatory syndrome (cHIS) [28]. As part of this study, we independently evaluated the relevance of the cHIS scoring system and determined the best cut-off in our cohort.

We defined clinical outcome as the length of hospitalization, the incidence of mechanical ventilation (including invasive and noninvasive ventilation), and mortality. We recorded the clinical outcomes with a follow-up of 90 days from hospital admission.

Additionally, 1513 non-COVID-19 adult patients, whose plasma zinc concentrations were previously measured in our hospital, were randomly selected (regardless of gender or comorbidity during 2018) to constitute the control group. Only age and plasma zinc concentrations were accessible for these patients.

\subsection{Statistical analysis}

Data were analyzed using Stata version 13 (StataCorp., USA). The distribution of both groups was assessed using percentages and proportions.

cHIS score was validated using the area under the receiver operating characteristic curve (AUROC) for discrimination and Hosmer-Lemeshow goodness of fit test for calibration. A cHIS cut-off, which would correspond to a CRS, was calculated using Youden's J statistic. The association of plasma zinc concentration and CRS was then investigated using a Mann-Whitney $U$ test.

The association between outcome and plasma zinc concentration was controlled with a spearman correlation test for the length of stay and logistic regression for both mechanical ventilation and mortality.

The plasma zinc levels were compared between the COVID-19 cohort and control group applying a Mann-Whitney $U$ test, Kruskal-Wallis test followed by Dunn's post hoc test and age-adjusted Logistic regression. The variable age was further included in the model since age could be a confounder [29].

The $p<0.05$ was set to define the significance level followed by a Bonferroni adjustment when required.

\section{Results}

\subsection{Baseline characteristics and patients' outcomes}

One hundred and thirty-nine eligible patients were included in the COVID-19 cohort. The patient characteristics and laboratory assessments are summarized in Table 1 and Fig. 1.

The majority of patients were male $(91 / 139,65 \%)$, and the median age of the cohort was 65 years (IQR $=54,77)$. Ninety-six patients $(69 \%)$ had two or more comorbidities. The median BMI was $27 \mathrm{~kg} / \mathrm{m}^{2}$ and 93/ $135(69 \%)$ patients had a BMI $>25 \mathrm{~kg} / \mathrm{m}^{2}$, among which $41 / 135(30 \%)$ patients were obese (BMI $\left.>30 \mathrm{~kg} / \mathrm{m}^{2}\right)$. The median time from symptoms onset to inclusion was eight days (IQR, 6 to 12).

The median worst daily WHO severity score was 4 (IQR $=4,6$ ). Forty-two patients (30\%) required mechanical ventilation or deceased during their hospitalization (WHO score $>5$, severe COVID-19) [27].

Nineteen patients (14\%) were admitted to the intensive care unit (ICU) during their hospital stay. Twenty patients (14\%) deceased within 90 days of admission, of whom seven (35\%) had been admitted to the ICU.

\subsection{Zinc status and its association with patients' clinical outcomes}

The median plasma zinc concentration in our cohort was $57 \mu \mathrm{g} / \mathrm{dL}$ $(\mathrm{IQR}=45,67)$ compared to a median of $74 \mu \mathrm{g} / \mathrm{dL}(\mathrm{IQR}=64,84)$ in the 
Table 1

Clinical and laboratory characteristics of 139 PCR-confirmed and hospitalized COVID-19 patients.

\begin{tabular}{|c|c|c|}
\hline $\begin{array}{l}\text { Clinical and laboratory characteristics of study } \\
\text { cohort }(\mathrm{N}=139) \text {. }\end{array}$ & $\begin{array}{l}\text { No. } \\
\text { Available }\end{array}$ & Value \\
\hline Age (yr), median [IQR] & 139 & $65[54-77]$ \\
\hline Gender - N (\%) & 139 & \\
\hline Female & & $48(34)$ \\
\hline Male & & $91(65)$ \\
\hline BMI $\left(\mathrm{kg} / \mathrm{m}^{2}\right)$, median [IQR] & 135 & $\begin{array}{l}27,2 \\
{[23-48]}\end{array}$ \\
\hline Overweight (BMI $\geq 25$ ) & & $52(38)$ \\
\hline Obese (BMI $\geq 30$ ) & & $41(30)$ \\
\hline \multicolumn{3}{|l|}{ Smoking status, N (\%) } \\
\hline Never & & $97(70)$ \\
\hline Former & & $35(25)$ \\
\hline Current & & $7(5)$ \\
\hline Number of coexisting conditions, $\mathrm{N}(\%)$ & 139 & \\
\hline 0 & & $5(4)$ \\
\hline 1 & & $19(14)$ \\
\hline 2 & & $19(14)$ \\
\hline$>2$ & & $96(69)$ \\
\hline Coexisting conditions, $\mathrm{N}(\%)$ & 139 & \\
\hline Hypertension & & $90(65)$ \\
\hline Diabetes & & $50(36)$ \\
\hline Hypercholesterolemia & & $54(39)$ \\
\hline Obstructive sleep apnea syndrome & & $11(8)$ \\
\hline Obstructive lung diseases & & $25(18)$ \\
\hline Coronary artery disease & & $19(14)$ \\
\hline Congestive heart failure & & $23(16)$ \\
\hline Atrial fibrillation & & $14(10)$ \\
\hline Chronic liver disease & & $4(3)$ \\
\hline Chronic kidney disease & & $31(22)$ \\
\hline Malign neoplasm & & $9(6)$ \\
\hline Immune suppression & & $25(18)$ \\
\hline Neurological nonvascular & & $12(9)$ \\
\hline Stroke & & $8(6)$ \\
\hline $\begin{array}{l}\text { Days with symptoms before inclusion, median } \\
\text { [IQR] }\end{array}$ & 131 & $8[6-12]$ \\
\hline $\begin{array}{l}\text { Days of hospitalization before inclusion, median } \\
\text { [IQR] }\end{array}$ & 139 & $1[1-2]$ \\
\hline COVID-19 medical therapy before inclusion & 139 & $108(78)$ \\
\hline Hydroxychloroquine & & $9(6)$ \\
\hline Dexamethasone & & $99(71)$ \\
\hline Severity of COVID-19 (worst WHO score) & 139 & \\
\hline Median WHO score [IQR] & & $4[4-6]$ \\
\hline With score $>5$ (severe disease), $\mathrm{N}(\%)$ & & $42(30)$ \\
\hline cHIS score at inclusion & 134 & \\
\hline Median [IQR] & & $2[1-2]$ \\
\hline With cHIS score $\geqq 2$, N (\%) & & $74(55)$ \\
\hline Plasma zinc (Normal range: $[80-120 \mu g / d L]$ ) & 139 & \\
\hline Median level [IQR] & & 57 [45-67] \\
\hline With zinc deficiency, $\mathrm{N}(\%)$ & & $133(96)$ \\
\hline
\end{tabular}

Abreviations: $I Q R=$ interquartile range, $C I=$ confidence interval, $O R=$ odds ratio.

control group. A Mann-Whitney $U$ test confirms that this difference is significant $(p<0.001)$.

Markedly, the absolute majority of COVID-19 inpatients (96\%) were zinc deficient $(<80 \mu \mathrm{g} / \mathrm{dL})$.

We observed that the plasma zinc concentration is weakly but significantly correlated (rho $=-0.19 ; p=0.022$ ) with the length of hospital stay (Fig. 2). However, the plasma zinc concentration was not significantly associated with the risk of mortality or morbidity (Tables 2a and 2b). Still, the median plasma zinc levels were systematically lower in mechanically ventilated compared to non-mechanically ventilated COVID-19 cases $(-5.23 \mu \mathrm{g} / \mathrm{dL} ; 95 \%$ confidence interval [CI] $=-10.57,0.13 ; p=0.058$ ), and in dead compared to surviving participants (-6. $68 \mu \mathrm{g} / \mathrm{dL} ; 95 \% \mathrm{CI}=-13.68,0.33 ; p=0.065)$.

\subsection{External validation of the cHIS score as the measure of inflammatory status}

The AUROC for mechanical ventilation and mortality was 0.687 and
0.674, respectively, which indicates a lack of discrimination capacity (AUC $\leq 0.7$ ) [30]. Nevertheless, according to the results of the HosmerLemeshow goodness of fit test [30], the score demonstrated a good calibration ( $p$-value $>0.05$ ), with $p$-values of 0.681 and 0.649 for mechanical ventilation and mortality, respectively.

Next, we aimed to identify a cHIS cut-off corresponding to CRS to define a binary (low- and high-risk for poor outcome) patient group regarding inflammatory status. Our data corroborate with the cut-off suggested by Webb et al. [28] demonstrating the best performance (Youden's J statistic closest to one) at a threshold of $\geqq 2$ [31].

In our cohort, 74/134 (55\%) patients had a cHIS score $\geqq 2$. Among patients with cHIS score $\geqq 2$, greater proportions of the patients needed mechanical ventilation $(30 / 74,40 \%)$ compared to patients with cHIS scores $<2(11 / 60,18 \%, p=0.006)$. Likewise, a higher mortality was observed $(16 / 74,22 \%)$ in the patient group with cHIS score $\geqq 2$ vs. cHIS scores $<2(4 / 60,7 \%, p=0.013)$.

Although our results support the association of CRS with severe COVID-19 and mortality, unlike Webb et al., we could not demonstrate an excellent discrimination capacity of the cHIS score in our cohort [28]. In contrast to that study, most of our patients $(108 / 139,78 \%)$ had received immunomodulatory therapies just before the laboratory evaluation as part of our standard of care and based upon WHO guidelines and findings of the Recovery trial [32]. Of note, Dexamethasone, part of our standard of care, reduces CRS and improves the outcome while inevitably lowers the cHIS score [32]. The cHIS score is probably more appropriate to identify high-risk patients in ambulatory practice or among the hypoxemic and dexamethasone-naïve patients.

\subsection{Association of plasma zinc concentration and cHIS score}

We were not able to show a significant association between plasma zinc levels and the development of CRS (defined as cHIS score $\geqq 2$ ) in our cohort.

The median concentration of plasma zinc was lower in patients with CRS compared to those without CRS $(-5 \mu \mathrm{g} / \mathrm{dL} ; 95 \% \mathrm{CI}=-10.5,0.051 ; p$ $=0.048$ ). However, with stringent and conservative Bonferroni adjustment, the results were not statistically significant.

\section{Discussion and limitations of the study}

The small sample size is the major limitation. Additionally, as the majority of the patients were zinc deficient, no room was left to compare zinc deficient and non-existing zinc sufficient patients. This could be partly attributable to the fact that the median age of our patient cohort is 65 years, which overlaps with the population at risk for zinc deficiency [33]. Moreover, our selection criteria included inpatients with PCRconfirmed SARS-Cov-2, which excluded ambulatory patients and those who did not require hospitalization. It is increasingly known that exacerbation and severity of illness begin within one week of symptom onset $[34,35]$. Therefore, it is not surprising that the median time from symptom onset to inclusion was eight days in our cohort. We thus realize that our selection criteria led to the enrichment of patients with more severe disease and at higher risk of poor outcomes, especially the elderly group.

Moreover, we compared the plasma zinc concentrations of COVID-19 patients with a retrospective control group of 1513 non-COVID-19 patients, selected regardless of gender or comorbidity during 2018. Except for age and plasma zinc concentration, we did not have access to complete individual data.

Correspondingly, the median age of patients in the COVID-19 cohort was higher (65 years) than the control population (49 years). However, we found that after applying age-adjusted logistic regression, the zinc concentrations and COVID-19 still remained in a negative relationship $(\mathrm{OR}=0.92 ; 95 \% \mathrm{CI}=0.91,0.94 ; p<0.001)$. Additionally, we stratified plasma zinc levels according to the mutually existing age groups in the COVID-19 cohort and the non-COVID19 control group (30-44, 45-59, 


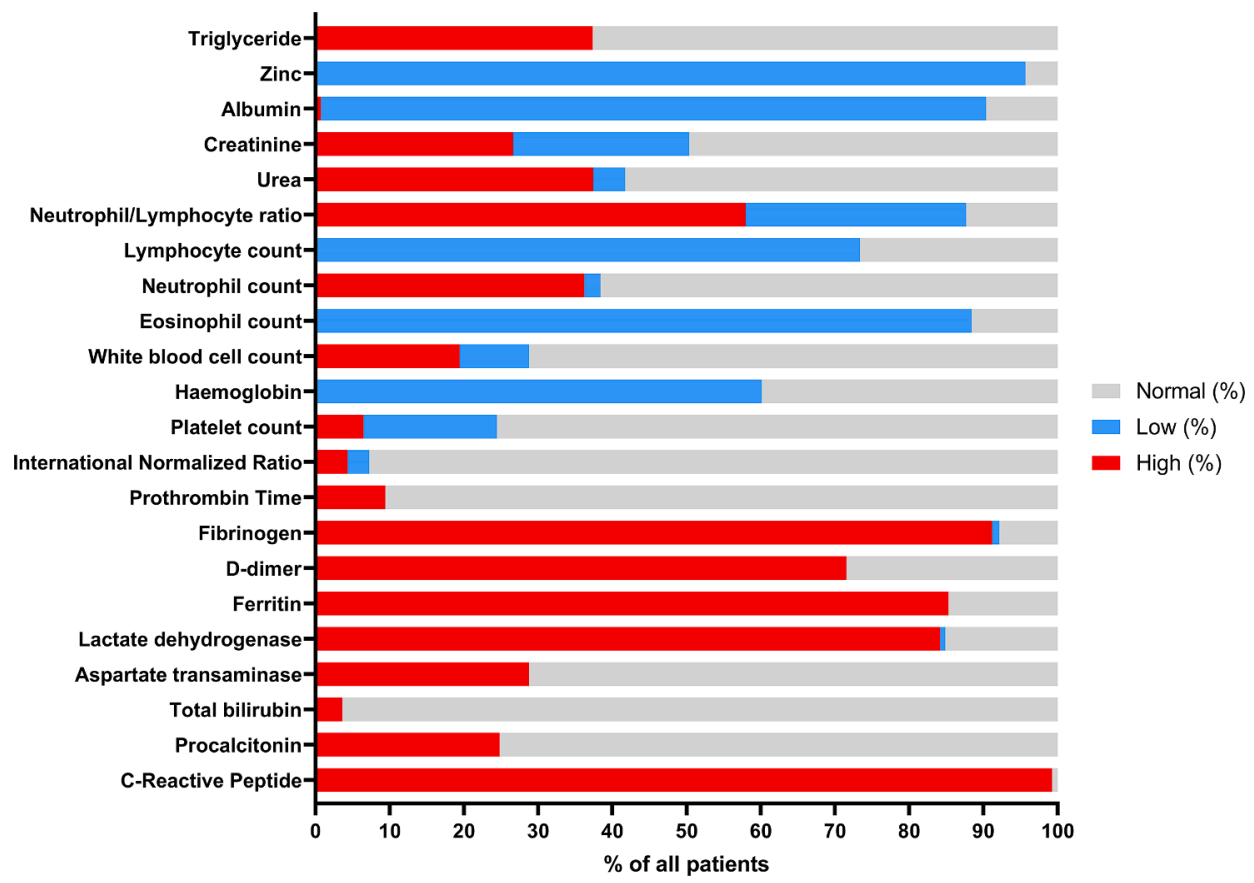

Fig. 1. Laboratory results are displayed as the percentage of deficient- versus sufficient patients in a cohort of 139 PCR confirmed and hospitalized COVID19 patients.

\section{Zinc vs. Hospital days}

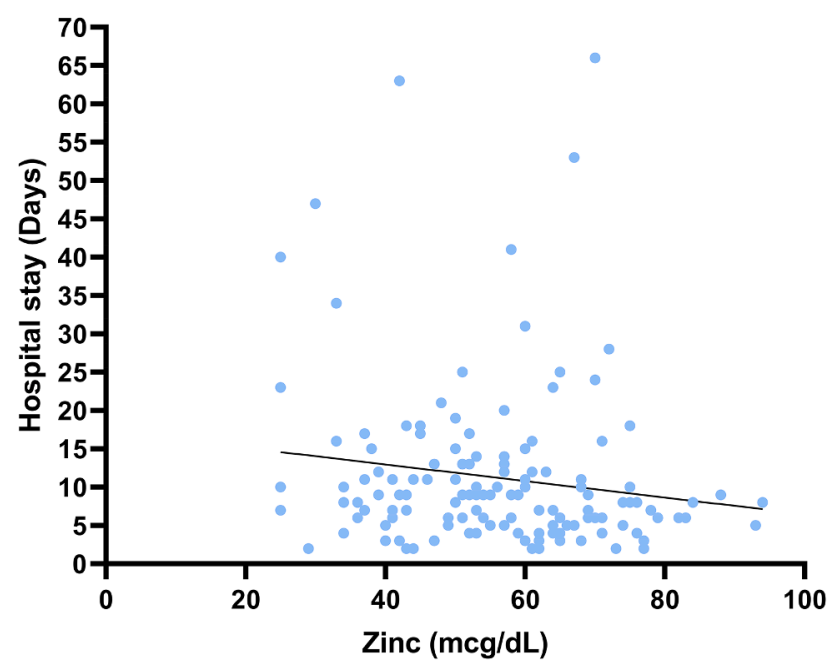

Fig. 2. Correlation curve illustrating the reverse association (weak) between zinc plasma concentration and the length of hospital stay in confirmed COVID19 inpatients (rho $=-0.19 ; p=0.022$ ).

Table 2a

Outcomes (defined as length of stay, mechanical ventilation ICU admission or deased) at 90-days for 139 PCR confirmed, hospitalized COVID-19 patients.

\begin{tabular}{ll}
\hline Outcomes at 90-days $(\mathbf{N}=\mathbf{1 3 9})$ & Value \\
\hline Length of hospital stay (days), median [IQR] & $8[5-13]$ \\
Mechanical ventilation, N (\%) & $42(30)$ \\
ICU admission, N (\%) & $19(14)$ \\
Deceased, N (\%) & $20(14)$ \\
\hline
\end{tabular}

Abreviations: IQR $=$ interquartile range, $\mathrm{CI}=$ confidence interval, $\mathrm{OR}=$ odds ratio.
Table 2b

Association of outcomes and plasma zinc level.

\begin{tabular}{ll}
\hline $\begin{array}{l}\text { Association of outcomes and plasma zinc } \\
\text { level } \mathbf{( N}=\mathbf{1 3 9})\end{array}$ & Value \\
\hline Lenght of hospital stay and zinc level & Correlation coefficien $(95 \% \mathrm{CI})=$ \\
& $-0.19(-0.35-0.03), p=0.022^{*}$ \\
Mechanical ventilation and zinc level & OR $(95 \% \mathrm{CI})=0.98(0.95-1.00), p$ \\
& $=0.058^{* *}$ \\
Mortality and zinc level & OR $(95 \% \mathrm{CI})=0.97(0.94-1.00), p$ \\
& $=0.0655^{* * *}$ \\
\hline "Spearman correlation & \\
$*$ Logistic regression &
\end{tabular}

and $60+$ years old). The median plasma zinc concentration in all three age-stratified groups of the COVID-19 cohort was significantly lower than in the corresponding control groups according to multiple comparisons across all age-stratified groups $(55,59,57 \mu \mathrm{g} / \mathrm{dL}$ vs. $73,73,73$ $\mu \mathrm{g} / \mathrm{dL}$, Kruskal-Wallis test followed by Dunn's post hoc test: $p=0.0006$, $p<0.001$ and $p<0.001$ respectively).

\section{Conclusions}

We report that an absolute majority of hospitalized COVID-19 patients are zinc deficient. We find a weak (reverse) correlation between plasma zinc concentration and the length of hospital stay, but not with mortality or morbidity. We independently validated cHIS as a score of COVID-19 severity, but we found no significant association between zinc plasma concentration and cHIS among patients who are almost all zinc deficient. As such, our findings do not support the role of zinc as a robust prognostic factor among hospitalized COVID-19 patients. We recommend zinc to be measured prospectively in a larger, non-COVID-19 population to assess the incidence of the disease and tissue-damaging CRS occurrence and related outcomes in zinc-deficient versus zincsufficient individuals.

Future studies should include both individuals who are freshly diagnosed with COVID-19 and ambulatory COVID-19 patients. As such, the prevalence of zinc deficiency can be determined at the disease onset, 
and the differential characteristics of those with hypozincemia can be unraveled. Additionally, consecutive zinc measurements throughout disease development can enable a more detailed analysis of the correlation between zinc levels, disease progression, and outcomes.

\section{Ethics approval and consent to participate}

Informed consent was obtained from all participants. The study was approved by the ethics committee of Erasme Hospital, EC identifier P2020/261.

\section{Availability of data and materials}

The datasets used and/or analysed during the current study are available from the corresponding author on reasonable request.

\section{CRediT authorship contribution statement}

Gil Verschelden: Writing - original draft, Conceptualization, Methodology, Investigation, Data curation, Formal analysis. Maxim Noeparast: Writing - original draft, Conceptualization, Methodology, Formal analysis, Validation. Maryam Noparast: Writing - review \& editing, Methodology, Formal analysis, Validation. Mathijs Christiaan Goossens: Writing - review \& editing, Methodology, Formal analysis, Validation. Maïlis Lauwers: Resources. Frédéric Cotton: Resources. Charlotte Michel: Resources. Cleo Goyvaerts: Writing - review \& editing, Methodology. Maya Hites: Writing - review \& editing, Supervision.

\section{Declaration of Competing Interest}

The authors declare that they have no known competing financial interests or personal relationships that could have appeared to influence the work reported in this paper.

\section{Acknowledgements}

Special thanks to Prof.Dr. Frédérique Jacobs (Université Libre de Bruxelles, Erasme Hospital).

\section{References}

[1] D.E. Leisman, et al., Cytokine elevation in severe and critical COVID-19: a rapid systematic review, meta-analysis, and comparison with other inflammatory syndromes, Lancet. Respir. Med. 8 (12) (Dec. 2020) 1233-1244, https://doi.org/ 10.1016/S2213-2600(20)30404-5.

[2] L.Y.C. Chen, R.L. Hoiland, S. Stukas, C.L. Wellington, M.S. Sekhon, Confronting the controversy: interleukin- 6 and the COVID-19 cytokine storm syndrome, Eur. Respir. J. 56 (4) (Oct. 2020) 2003006, https://doi.org/10.1183/13993003.030062020.

[3] J. Shang, et al., Structural basis of receptor recognition by SARS-CoV-2, Nature 581 (7807) (2020) 221-224, https://doi.org/10.1038/s41586-020-2179-y.

[4] I. Hamming, W. Timens, M.L.C. Bulthuis, A.T. Lely, G.J. Navis, H. van Goor, Tissue distribution of ACE2 protein, the functional receptor for SARS coronavirus. A first step in understanding SARS pathogenesis, J. Pathol. 203 (2) (Jun. 2004) 631-637, https://doi.org/10.1002/path.1570.

[5] K. C. Xin Zou Jiawei Zou, Peiyi Han, Jie Hao, Zeguang Han, "Single-cell RNA-seq data analysis on the receptor ACE2 expression reveals the potential risk of different human organs vulnerable to 2019-nCoV infection. Front. Med., vol. 14, no. 2. pp. 185-192, [Online]. Available: https://journal.hep.com.cn/fmd.

[6] M. Merad, J.C. Martin, Pathological inflammation in patients with COVID-19: a key role for monocytes and macrophages, Nat. Rev. Immunol. 20 (6) (2020) 355-362, https://doi.org/10.1038/s41577-020-0331-4.

[7] C.E. Barkauskas, et al., Type 2 alveolar cells are stem cells in adult lung, J. Clin. Invest. 123 (7) (Jul. 2013) 3025-3036, https://doi.org/10.1172/JCI68782.

[8] A.M. Olajuyin, X. Zhang, H.-L. Ji, Alveolar type 2 progenitor cells for lung injury repair, Cell Death Discov. 5 (1) (2019) 63, https://doi.org/10.1038/s41420-0190147-9.
[9] P. Verdecchia, C. Cavallini, A. Spanevello, F. Angeli, The pivotal link between ACE2 deficiency and SARS-CoV-2 infection, Eur. J. Intern. Med. 76 (Jun. 2020) 14-20, https://doi.org/10.1016/j.ejim.2020.04.037.

[10] M.P. Salgo, COVID-19: Zinc and Angiotensin-Converting Enzyme 2 (ACE2) Deficiencies as Determinants of Risk and Severity of Disease: A Narrative Review, Infect. Dis. Ther. 10 (3) (Sep. 2021) 1215-1225, https://doi.org/10.1007/s40121021-00478-8.

[11] S.A. Read, S. Obeid, C. Ahlenstiel, G. Ahlenstiel, The Role of Zinc in Antiviral Immunity, Adv. Nutr. 10 (4) (Apr. 2019) 696-710, https://doi.org/10.1093/ advances/nmz013.

[12] A.S. Prasad, Zinc in human health: effect of zinc on immune cells, Mol. Med. 14 (5-6) (2008) 353-357, https://doi.org/10.2119/2008-00033.Prasad.

[13] P. Towler, et al., ACE2 X-ray structures reveal a large hinge-bending motion important for inhibitor binding and catalysis, J. Biol. Chem. 279 (17) (Apr. 2004) 17996-18007, https://doi.org/10.1074/jbc.M311191200.

[14] Noeparast, A.; Verschelden, G.; Degeyter, D.; Marco, M.; Goyvaerts, C.; Hites, M. In Light of the SARS-CoV-2 Pandemic: Revisit of the Evidence Associating Zinc and Anti-viral Response. Preprints 2020, "In Light of the SARS-CoV-2 Pandemic: Revisit of the Evidence Associating Zinc and Anti-viral Response. Preprints, vol. 2020040094, 2020.

[15] I. Wessels, B. Rolles, L. Rink, The Potential Impact of Zinc Supplementation on COVID-19 Pathogenesis, Front. Immunol. 11 (2020) 1712, https://doi.org/ 10.3389/fimmu.2020.01712.

[16] S.H. Asl, S. Nikfarjam, N. Majidi Zolbanin, R. Nassiri, R. Jafari, Immunopharmacological perspective on zinc in SARS-CoV-2 infection, Int. Immunopharmacol. 96 (2021), https://doi.org/10.1016/j.intimp.2021.107630.

[17] F. Boudreault et al., Zinc deficiency primes the lung for ventilator-induced injury, JCI insight 2(11) (2017), doi: 10.1172/jci.insight.86507.

[18] N.N. Gomez, M.S. Ojeda, M.S. Gimenez, Lung lipid composition in zinc-deficient rats, Lipids 37 (3) (Mar. 2002) 291-296, https://doi.org/10.1007/s11745-0020893-3.

[19] D.L. Knoell, et al., Zinc deficiency increases organ damage and mortality in a murine model of polymicrobial sepsis, Crit. Care Med. 37 (4) (Apr. 2009) 1380-1388, https://doi.org/10.1097/CCM.0b013e31819cefe4.

[20] I. Wessels, et al., Zinc supplementation ameliorates lung injury by reducing neutrophil recruitment and activity, Thorax 75 (3) (Mar. 2020) 253-261, https:// doi.org/10.1136/thoraxjnl-2019-213357.

[21] I. Cakman, H. Kirchner, L. Rink, Zinc supplementation reconstitutes the production of interferon-alpha by leukocytes from elderly persons, J. Interferon Cytokine Res. 17 (8) (Aug. 1997) 469-472, https://doi.org/10.1089/jir.1997.17.469.

[22] C. Kitabayashi, et al., Zinc suppresses Th17 development via inhibition of STAT3 activation, Int. Immunol. 22 (5) (May 2010) 375-386, https://doi.org/10.1093/ intimm/dxq017.

[23] A.S. Prasad, et al., Zinc supplementation decreases incidence of infections in the elderly: effect of zinc on generation of cytokines and oxidative stress, Am. J. Clin. Nutr. 85 (3) (Mar. 2007) 837-844, https://doi.org/10.1093/ajcn/85.3.837.

[24] C.P. Wong, N.A. Rinaldi, E. Ho, Zinc deficiency enhanced inflammatory response by increasing immune cell activation and inducing IL6 promoter demethylation, Mol. Nutr. Food Res. 59 (5) (May 2015) 991-999, https://doi.org/10.1002/ mnfr.201400761.

[25] P. Mehta, D.F. McAuley, M. Brown, E. Sanchez, R.S. Tattersall, J.J. Manson, COVID-19: consider cytokine storm syndromes and immunosuppression, Lancet (London, England). England, Mar. (2020), https://doi.org/10.1016/S0140-6736 (20)30628-0.

[26] I.J. Davies, M. Musa, T.L. Dormandy, Measurements of plasma zinc. I. In health and disease, J. Clin. Pathol. 21 (3) (May 1968) 359-363, https://doi.org/10.1136/ jcp.21.3.359.

[27] "A minimal common outcome measure set for COVID-19 clinical research.," Lancet. Infect. Dis., vol. 20, no. 8, pp. e192-e197, Aug. 2020, doi: 10.1016/S14733099(20)30483-7.

[28] B.J. Webb, et al., Clinical criteria for COVID-19-associated hyperinflammatory syndrome: a cohort study, Lancet. Rheumatol. 2 (12) (Dec. 2020) e754-e763, https://doi.org/10.1016/S2665-9913(20)30343-X.

[29] Á.J.R. Cabrera, Zinc, aging, and immunosenescence: an overview, Pathobiol. Aging Age Relat. Dis. 5 (Feb. 2015) 25592, https://doi.org/10.3402/pba.v5.25592.

[30] D.W. Hosmer, S. Lemeshow, Applied Logistic Regression Second Edition. 2004.

[31] W.J. Youden, Index for rating diagnostic tests, Cancer 3 (1) (Jan. 1950) 32-35, https://doi.org/10.1002/1097-0142(1950)3:1<32::aid-cncr2820030106>3.0.co; 2-3.

[32] P. Horby, et al., Dexamethasone in Hospitalized Patients with Covid-19, N. Engl. J. Med. 384 (8) (Feb. 2021) 693-704, https://doi.org/10.1056/NEJMoa2021436.

[33] N.M. Lowe, K. Fekete, T. Decsi, Methods of assessment of zinc status in humans: a systematic review, Am. J. Clin. Nutr. 89 (6) (Jun. 2009) 2040S-2051S, https://doi. org/10.3945/ajcn.2009.27230G.

[34] J. Liu, et al., Clinical and radiological changes of hospitalised patients with COVID19 pneumonia from disease onset to acute exacerbation: a multicentre paired cohort study, Eur. Radiol. 30 (10) (Oct. 2020) 5702-5708, https://doi.org/ 10.1007/s00330-020-06916-4.

[35] D.A. Berlin, R.M. Gulick, F.J. Martinez, Severe Covid-19, N. Engl. J. Med. 383 (25) (Dec. 2020) 2451-2460, https://doi.org/10.1056/NEJMcp2009575. 